\title{
Selfduality and Topological-Like Properties of Lattice Gauge Field Theories. A Proposal
}

\author{
P. Cotta-Ramusino* and G. Dell'Antonio \\ Institut für theoretische Physik, Freie Universität Berlin, D-1000 Berlin 33, and Istituto Matematico \\ G. Castelnuovo, Università di Roma, I-00100 Roma, Italy
}

\begin{abstract}
We introduce for lattice gauge theories an analogue of the Pontrjagin index and a notion of "selfduality" and "antiselfduality". Selfdual and antiselfdual configurations on the lattice have much of the same properties (with some remarkable differences) as the corresponding configurations on the continuum, to which they converge when the lattice spacing goes to zero.
\end{abstract}

\section{Introduction}

In the past few years much effort has been put on the study of the topological properties of gauge fields. Particular emphasis has been placed on the use of topological invariants and of relevant techniques from differential and algebraic geometry to classify the solutions of the Yang-Mills equations which correspond to absolute minima of the action functional [1-8].

The ultimate interest in gauge theories from the point of view of elementary particle physics is of course the solution of the quantum Yang-Mills equations. The hope to be able to use also for gauge theories an approach trough (an Euclidean version of) path integral techniques makes it generally felt that one could benefit from a thorough understanding of the set of classical solutions, especially the absolute minima of the action, from a topological point of view.

The fact that the (yet to be constructed) measure on the space of connections (Yang-Mills potentials) is expected to give measure zero to the set of differentiable potentials (while the classical solutions are twice differentiable almost everywhere) is probably not a serious objection as one can see from the analogous problem in Quantum Mechanics.

On the other hand a mathematical theory of integration on function spaces does not exist at present for gauge theories; in view of the experience accumulated

* On leave of absence from Istituto di Fisica dell'Università di Milano, Via Celoria 16, 20133 Milano, Italy.

Research partially supported by Consiglio Nazionale delle Ricerche and Fondazione A. Della Riccia 
in the recent studies of scalar non linear field theories $[9,10]$, one is led to consider as a suitable intermediate step the study of a "lattice" approximation of the Euclidean version of the theory. By considering only points of a suitable (usually cubic) lattice and functions with values in a (compact) Lie Group rather than in the corresponding Algebra, this approximation exploits the advantage of working with measures on compact sets, and has had some preliminary success [11-13].

These very approximations seem however at first sight to constitute a serious drawback from a geometric point of view. One seems to have lost the topological and differential-geometrical structure which had been most useful in the treatment of the classical Yang-Mills fields in the continuum.

In this note, we want to suggest a possible partial remedy to this situation, by introducing in the lattice approximation some structures which are "gauge invariant" and reproduce, in the continuum limit, the topological invariants of the continuum case. In particular, we shall define a "Pontrjagin density" in the lattice and a notion of selfduality and antiselfduality.

It will turn out that these entities can be defined in a rather natural way, and have many of the properties of the corresponding quantities defined for fiber bundles over manifolds - to which they converge when the lattice spacing goes to zero - although they lack of course their "intrinsic" meaning.

We expect that the quantities we introduce here will be useful to study the leading contributions of lattice quantum gauge theories in the strong coupling limit, and possibly also to study the convergence to the continuum limit.

We shall not pursue the latter aspect in this paper.

\section{A Brief Review of the Continuum Case}

We shall recall briefly some well known results of Yang-Mills theories on $E^{4}$ (Euclidean four dimensional space) to establish our notation and to provide motivation for our approach on the lattice.

Let $G$ be a simple, compact, non abelian Lie group ( $G$ will be taken to be $\mathrm{SU}(n)$, $\mathrm{SO}(n)$ or $\mathrm{Sp}(n)$ in most of what follows) and $\mathfrak{g}$ be the corresponding Lie algebra.

In this paper, when there is no danger of confusion we shall denote by the same symbols $G, \mathfrak{g}$ any unitary finite dimensional faithful representation of the group and the corresponding representation of the Lie algebra.

We recall that, if $B$ and $C$ are matrix-valued forms on a manifold $M$, which can be written as $B=\mathscr{B} \otimes f, C=\mathscr{C} \otimes g$ with $\mathscr{B}, \mathscr{C}$ complex matrices and $f, g \in\left(T^{*} M\right)$, then by definition:

$$
\begin{aligned}
B \wedge C & =:(\mathscr{B} \cdot \mathscr{C}) \otimes(f \wedge g) \\
{[B, C] } & =:[\mathscr{B}, \mathscr{C}] \otimes(f \wedge g) .
\end{aligned}
$$

These definitions extend to all matrix-valued forms by linearity.

Suppose now that $M$ is the one-point compactification of $E^{4}$, and let $P(M, G)$ be a Principal Fibre bundle over $M$ with group $G$, connection form $\omega$ and curvature form $\Omega=d \omega+\frac{1}{2}[\omega, \omega]$.

In local coordinates we set $A=: \phi^{*} \omega$ and $F=: \phi^{*} \Omega$, where $\phi$ is a (local) cross section $\phi: M \rightarrow P$. $A$ is called the Yang-Mills potential, $F$ is the Yang-Mills Field and $\phi$ is called also a "gauge". 
$\Omega$ is an horizontal 2-form of type $\operatorname{Ad} G$, so it determines a unique 2-form on $M$ taking its values in the bundle $P \times{ }_{G}$ g associated to $P$ and to the adjoint representation. Then, if $*$ is the Hodge operator, $\operatorname{Tr}\left({ }^{*} F \wedge F\right)$ and $\operatorname{Tr}(F \wedge F)$ can be considered as ordinary 4 -forms on $M$. by:

The Pontrjagin index (i.e. minus the integral of the $2^{\text {nd }}$ Chern class) is defined

$$
K=-\frac{1}{8 \pi^{2}} \int_{M} \operatorname{Tr}(F \wedge F)
$$

while the "action" (i.e. the square of the norm of the curvature) is:

$$
S=-\frac{1}{4} \int_{M} \operatorname{Tr}(* F \wedge F)
$$

Since $M$ is the one point compactification of $E^{4}$, one can view $E^{4}$ as a chart for $M$ (with the identification map). In the local coordinates of this chart one has:

$$
\begin{aligned}
& F=\frac{1}{2} \sum_{\alpha} \sum_{\mu, v=1}^{4} F_{\mu \nu}^{\alpha}(x) d x^{\mu} \wedge d x^{v} e_{\alpha}, \\
& A=\sum_{\alpha} \sum_{\mu=1}^{4} A_{\mu}^{\alpha}(x) d x^{\mu} e_{\alpha},
\end{aligned}
$$

where $e_{\alpha}$ is a basis in $\mathfrak{g}$.

Setting $F_{\mu \nu}=\sum_{\alpha} F_{\mu \nu}^{\alpha} e_{\alpha}, A_{\mu}=\sum_{\alpha} A_{\mu}^{\alpha} e_{\alpha}$, we have the usual relation:

$$
F_{\mu v}(x)=\partial_{\mu} A_{v}(x)-\partial_{v} A_{\mu}(x)+\left[A_{\mu}(x), A_{v}(x)\right] \text {. }
$$

We shall consider only Yang-Mills fields with finite action integral (2.2), but remark that this restriction was already hidden in the statement that $\operatorname{Tr}\left({ }^{*} F \wedge F\right)$ can be defined as a form on $M$.

We have (with the Einstein convention):

$$
\begin{aligned}
& S=-\frac{1}{4} \int_{E^{4}} \operatorname{Tr}\left(F_{\mu \nu} F^{\mu \nu}\right) d^{4} x=: \int_{E^{4}} S(x) d^{4} x \\
& K=-\frac{1}{16 \pi^{2}} \int_{E^{4}} \operatorname{Tr}\left(F_{\mu \nu} F_{\varrho \sigma} \varepsilon^{\mu \nu \varrho \sigma}\right) d^{4} x=: \int_{E^{4}} K(x) d^{4} x,
\end{aligned}
$$

where $\varepsilon^{\mu \nu \rho \sigma}$ is the completely antisymmetric symbol.

Using the antisymmetry of $F_{\mu \nu}$ and the identity $\operatorname{Tr}(A B)=\operatorname{Tr}(B A)$ one obtains:

$$
S=-\frac{1}{2} \sum_{\mu<\nu} \int \operatorname{Tr} F_{\mu \nu}^{2} d^{4} x
$$




\section{Lattice Approximation: Preliminaries}

We follow essentially the notation of [13]. Denote by $Z_{\varepsilon}^{4}$ the cubic lattice in $E^{4}$ centered at the origin and with elementary linear lattice size $\varepsilon ; \Lambda$ will denote a bounded hypercube contained in $Z_{\varepsilon}^{4}$.

If $x$ is a generic lattice site, there exist integers $n_{i}, i=1, \ldots, 4$ such that $x=\left(n_{1} \varepsilon\right.$, $\left.n_{2} \varepsilon, n_{3} \varepsilon, n_{4} \varepsilon\right)$ where $n_{k} \varepsilon$ is the component of $x$ along the $k^{\text {th }}$ axis.

We shall denote by $x \pm \hat{k}$ the element of $Z_{\varepsilon}^{4}$ which has components $\left(n_{i} \pm \delta_{k i}\right) \varepsilon$, $i=1, \ldots, 4$.

Let $A_{\mu}(x)$ a Yang-Mills potential which we assume to behave at $|x| \rightarrow \infty$ as a pure gauge [i.e. $F_{\mu \nu}(x) \rightarrow 0$ when $|x| \rightarrow \infty$, sufficiently fast]. The segment (of length $\varepsilon$ ) $[x, x+\hat{k}]$ is by definition the (oriented) elementary link from $x$ to $x+\hat{k}$; we shall denote it by $(x ; \hat{k})$. To each elementary link we associate an element of $G$, defined as follows:

$$
f^{A}:(x ; \hat{k}) \rightarrow f^{A}(x ; \hat{k})=: \exp \left(\varepsilon A_{k}(x)\right) .
$$

Define a plaquette as an elementary square in $Z_{\varepsilon}^{4}$, enclosed by four elementary links. We denote by $(x ; \hat{\mu}, \hat{v})$ the plaquette in the $\hat{\mu}, \hat{v}$ plane, having $x$ as smallest point (in the natural order induced on $Z_{\varepsilon}^{4}$ by the direction of the four orthogonal axes). Plaquettes are oriented, so $(x ; \hat{\mu}, \hat{v}) \neq(x ; \hat{v}, \hat{\mu})$. To each elementary plaquette, we associate an element of $G$ according to the following rule:

$$
\begin{aligned}
& g^{A}:(x ; \hat{\mu}, \hat{v}) \rightarrow g^{A}(x ; \hat{\mu}, \hat{v}) \\
& \quad=: f^{A}(x ; \hat{\mu}) f^{A}(x+\hat{\mu} ; \hat{v})\left(f^{A}(x+\hat{v} ; \hat{\mu})\right)^{-1}\left(f^{A}(x ; \hat{v})\right)^{-1} .
\end{aligned}
$$

Since we deal only with finite-dimensional representations of $G$, one can easily check that $\operatorname{Tr} g^{A}$ is an analytic function of $\varepsilon$ at zero ; moreover, since $\operatorname{Tr} A=0 \forall A \in \mathfrak{g}$, one can compute:

$$
\operatorname{Tr} g^{A}(x ; \hat{\mu}, \hat{v})=\operatorname{Tr} \mathbb{1}+\frac{1}{2} \varepsilon^{4} \operatorname{Tr} F_{\mu v}(x)+O\left(\varepsilon^{5}\right) .
$$

It follows that, at least if $F_{\mu \nu}(x)$ is uniformly bounded in $E^{4}$ and decreases fast enough:

$$
\begin{aligned}
& \sum_{\text {Plaquettes }}\left(\operatorname{Tr} \mathbb{1}-\operatorname{Tr} g^{A}(P)\right)=\sum_{x \in Z_{\varepsilon}^{4}} \sum_{\mu, \nu=1, \ldots, 4} \operatorname{Tr}\left(-\frac{1}{4} \varepsilon^{4} F_{\mu \nu}^{2}\right)+O\left(\varepsilon^{5}\right) \\
& \stackrel{\varepsilon \rightarrow 0}{\longrightarrow}-\frac{1}{4} \int \operatorname{Tr}\left(\sum_{\mu \nu} F_{\mu \nu}^{2}\right) d^{4} x=S .
\end{aligned}
$$

In (3.4) we have therefore a natural candidate for the role of the action on the lattice.

We shall now introduce a lattice analogue of the Pontrjagin index. Notice that in the expression (2.7) for $K(x)$ one has terms of the form $F_{12}(x) F_{34}(x)$. This points to a structure in which one has a correlation between complementary planes (recall that we are in $E^{4}$ ). There is a natural way to introduce such correlation in a lattice.

We define biplaquette at $x$ a (unordered) pair of complementary plaquettes based at $x$ (i.e. such that for each of them $x$ is the lowest point).

For each $x \in Z_{\varepsilon}^{4}$ there are three distinct biplaquettes, which we shall denote $B P_{i}(x) i=1,2,3$. Each $B P_{i}(x)$ is made of two plaquettes $P_{i}^{\prime}(x)$ and $P_{i}^{\prime \prime}(x)$. The orientation of the plaquettes is indicated in Fig. 1. 


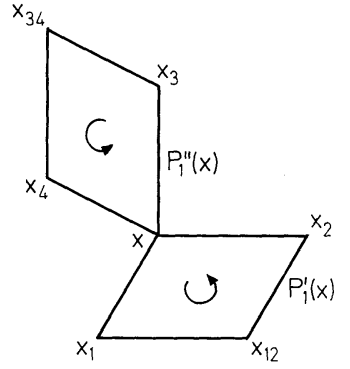

$B P_{1}(x)$

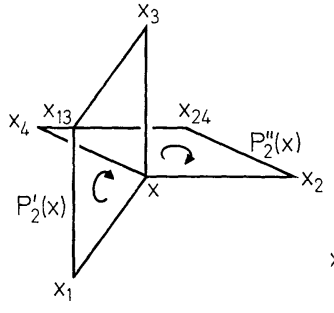

$\mathrm{BP}_{2}(\mathrm{x})$

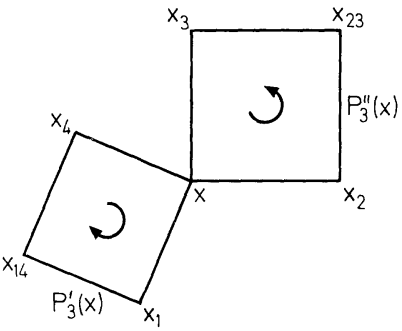

$\mathrm{BP}_{3}(x)$

Fig. 1.

To each biplaquette we associate an element of $G$ according to the rule:

$$
B P_{i}(x) \rightarrow h^{A}\left(B P_{i}(x)\right)=: g^{A}\left(P_{i}^{\prime}(x)\right) g^{A}\left(P_{i}^{\prime \prime}(x)\right) \text {. }
$$

Note that $h^{A}$ plays a role similar to that of a form of maximal degree. A straightforward computation leads to:

$$
\operatorname{Tr} \mathbb{1}-\operatorname{Tr} h^{A}\left(B P_{1}(x)\right)=-\frac{\varepsilon^{4}}{2} \operatorname{Tr}\left(F_{12}+F_{34}\right)^{2}+O\left(\varepsilon^{5}\right)
$$

and similar expressions for $h^{A}\left(B P_{2}(x)\right), h^{A}\left(B P_{3}(x)\right)$.

One has therefore:

$$
\begin{aligned}
\sum_{i=1}^{3}\left[\operatorname{Tr} \mathbb{1}-\operatorname{Tr} h^{A}\left(B P_{i}(x)\right)\right]= & -\frac{\varepsilon^{4}}{2} \operatorname{Tr}\left(\left(F_{12}+F_{34}\right)^{2}+\left(F_{13}+F_{24}\right)^{2}\right. \\
& \left.+\left(F_{14}+F_{23}\right)^{2}\right)+O\left(\varepsilon^{5}\right) .
\end{aligned}
$$

From (3.7) it follows, if $F$ is sufficiently smooth,

$$
\lim _{\varepsilon \rightarrow 0} \sum_{x \in Z_{\varepsilon}^{4}} \sum_{i=1}^{3}\left[\operatorname{Tr}\left(\mathbb{1}-h^{A}\left(B P_{i}(x)\right)\right]=S+2 \pi^{2} K .\right.
$$

In view of (3.1), (3.4), (3.8) it is reasonable to introduce the following definitions of action and Pontrjagin density of the lattice gauge configurations.

A configuration of a lattice gauge theory with local gauge group $G$ is a function $f$ from the elementary ordered links in $Z_{\varepsilon}^{4}$ to $G$.

The map $f$ satisfies the identity:

$$
f(x ; \hat{i})=(f(x+\hat{i} ;-\hat{i}))^{-1} \text {. }
$$

The function $f$ induces a map $g$ on the (oriented) plaquettes based at $x$, defined by:

$$
g(x ; \hat{i}, \hat{j})=f(x ; \hat{i}) f(x+\hat{i} ; \hat{j})(f(x+\hat{j} ; \hat{i}))^{-1}(f(x ; \hat{j}))^{-1} .
$$

We shall consider always any plaquette $P$ as "based" at its lowest point. On a Biplaquette $B P(x)$ composed of the two plaquettes $P^{\prime}(x)$ and $P^{\prime \prime}(x)$, the map $f$ induces a map $h$ through :

$$
h(B P(x))=: g\left(P^{\prime}(x)\right) g\left(P^{\prime \prime}(x)\right) .
$$


The order in which $P^{\prime}(x)$ and $P^{\prime \prime}(x)$ appear is the partial order induced on the pair of the plaquettes by the chosen lexicographic order of the orthogonal axes.

We define the action density $S_{L}(x)[13]$ and the Pontrjagin density $K_{L}(x)$ of a given configuration, in the following way:

$$
\begin{aligned}
S_{L}(x)= & : \sum_{\begin{array}{c}
\text { Plaquettes } \\
\text { based at } x
\end{array}}(\operatorname{Tr} \mathbb{1}-\operatorname{Re} \operatorname{Tr} g(P(x))), \\
K_{L}(x)= & : \frac{1}{2 \pi^{2}} \sum_{\begin{array}{c}
\text { Biplaquettes } \\
\text { based at } x
\end{array}}(\operatorname{Tr} \mathbb{1}-\operatorname{Re} \operatorname{Tr} h(B P(x))) \\
& -\frac{1}{2 \pi^{2}} \sum_{\begin{array}{c}
\text { Plaquettes } \\
\text { based at } x
\end{array}}(\operatorname{Tr} \mathbb{1}-\operatorname{Re} \operatorname{Tr} g(P(x))) .
\end{aligned}
$$

The action will then be $S_{L}=: \sum_{x \in Z_{\varepsilon}^{4}} S_{L}(x)$ and the Pontrjagin index $K_{L}=: \sum_{x \in Z_{\varepsilon}^{4}} K_{L}(x)$

We shall only consider configurations such that these sums are absolutely convergent.

Following [13] we define a gauge transformation as a map $\gamma$ from the lattice points into the group $G$.

Associated with a gauge transformation there is a transformation of the function $f: f \rightarrow f_{\gamma}$ with $f_{\gamma}(x ; \hat{k})=: \gamma(x) f(x ; \hat{k}) \gamma^{-1}(x+\hat{k})$.

It is obvious that both $S_{L}(x)$ and $K_{L}(x)$ are "gauge independent". We shall also call a gauge transformation a 0 -cochain on the lattice, a gauge configuration a 1-cochain on the lattice and a function which assign to every plaquette an element of $G$ a 2-cochain.

If we have a 0 -cochain $\gamma$ we can construct a 1-cochain defining: $f(x ; \hat{k})=: \gamma(x) \gamma^{-1}(x+\hat{k})$ and we shall write $f=d_{L} \gamma$.

If we have a 1-cochain $f$ the function $g$ defined on the plaquettes by the (3.9) is a 2-cochain and we shall write $g=d_{L} f$.

For each 0 -cochain $\gamma$ we have: $d_{L} d_{L} \gamma=e$ (i.e. the function which assign to every plaquette the identity of $G$ ).

It is also true that if a 1 -cochain $f$ is such that $d_{L} f=e$, then there exists a 0 cochain $\gamma$ such that $f=d_{L} \gamma$. That is every gauge configuration for which $g(P)=e \forall$ plaquette $P$ is gauge equivalent to the configuration which assign to every link the identity element of $G$.

To prove this statement choose with a suitable gauge transformation, equal to $e$ all the elements $f(l)$ if $l$ is contained in the line $x_{2}=x_{3}=x_{4}=0$ (see [13]). Then with a gauge transformation concentrated on the points of the two lines $x_{3}=x_{4}$ $=0 ; x_{2}= \pm \varepsilon$, fix equal to $e$ all the elements $f(l)$; where $l$ is one of the links $\left(\left(x_{1}, 0,0,0\right) ; \pm 2\right)$.

Since $g(P)=e \forall$ plaquette $P$, also the group elements associated to the links lying in the two lines $x_{3}=x_{4}=0 ; x_{2}= \pm \varepsilon$ must be equal to $e$.

Proceding further we can fix the group element equal to $e$ for all the links of the plane $x_{3}=x_{4}=0$; then successively for all the links of the three dimensional space $x_{4}=0$ and at last for all the links of $Z_{\varepsilon}^{4}$. 
So we can say that every 1-cocycle is a 1-coboundary (called also "trivial" or "pure gauge" configuration on the lattice).

Since $G$ is not abelian we are not able to extend the operator $d_{L}$ to the 2 cochains, so in general we have no 2-cocycles.

But we can in any case ask the question if a 2-cochain $g$ is a coboundary, that is if there exists a 1-cochain $f$ such that $g=d_{L} f$.

This problem will be discussed in Sect. 8 .

At the moment we note that if $g$ is a coboundary, then the 1-cochain $f$ such that $g=d_{L} f$ is unique (up to a gauge transformation).

More precisely if $f$ and $f^{\prime}$ are two 1 -cochains such that $d_{L} f=d_{L} f^{\prime}=g$, then as before, we can construct a gauge transformation $x \rightarrow \gamma(x)$ which transforms $f(x ; \hat{k})$ in $f^{\prime}(x ; \hat{k})$, but now $\gamma(x)$ will also satisfy the condition $\gamma(x) g(P(x)) \gamma^{-1}(x)=g(P(x))$ for every plaquette $P(x)$ based at $x$ and for every $x$; which "often" will imply that $\gamma(x)$ belongs to the center of $G$.

We will also use the word "gauge configuration" for 2-coboundaries.

As a concluding remark for this section, observe that for two gauge equivalent 2-coboundaries $g$ and $g^{\prime}$ we have conj. class $(g(P))=\operatorname{conj}$. class $\left(g^{\prime}(P)\right) \forall$ plaquette $P$, but the converse is not true, in the sense that conj. class $(g(P))=\operatorname{conj}$. class $\left(g^{\prime}(P)\right) \forall P$ does not imply that $g$ is gauge equivalent to $g^{\prime}$ unless $g(P)$ belongs to the center of $G, \forall P$ (or unless we consider configurations with values in an abelian group).

\section{Selfduality and Antiselfduality on the Lattice}

We recall that the Pontrjagin number defined on continuum configurations is useful in the study of quantum gauge theories for the following reasons, which are of course at the present moment of heuristic nature at most, once one has not found yet a convenient measure space.

In the (path space integral) euclidean formulation of quantum gauge theories one is led to write expressions of the form:

$$
\mathscr{E}(\mathscr{F})=\exp \left(-\frac{1}{\lambda} S(A)\right) \mathscr{F}(A) \mu(d A)
$$

where the measure $\mu$ is on a "suitable" space $\mathscr{M}$ of Potentials, $\mathscr{F}: \mathscr{M} \rightarrow \mathbb{R}$ is a gauge invariant functional, $S: \mathscr{M} \rightarrow \mathbb{R}^{+}$is the action functional, $\lambda$ is a parameter which will be taken sufficiently small.

One expects that the measure $\mu$ is carried by nowhere differentiable "potentials" $A$, so that the definition (2.4) for the action functional becomes meaningless, and a suitable definition should be given to $\exp \left(-\frac{1}{\lambda} S(A)\right) \mu(d A)$.

In spite of this, on the basis of the formal analogy with the quantum mechanical case and more generally with the treatment of some scalar (quantum) field theories in two or three space-time dimensions, one is led to believe that the leading (in $\lambda$ ) contribution to (4.1) comes from a neighborhood in $\mathscr{M}$ of those smooth potentials $A$ at which $S(A)$ reaches a minimum [recall that $S(A) \geqq 0$ for smooth $A$ 's]. 
In particular, to the leading order in $\lambda$, for sufficiently small, the integral $\mathscr{E}(\mathscr{F})$ is expected to be given by the sum (or integral) of $\exp \left(-\frac{1}{\lambda} S(A)\right)$ over those smooth configurations which minimize $S$. These "fields" are of course, at least formally, a solution of the Euler-Lagrange equations for the functional $S$.

The Pontrjagin index (2.5) takes only integer values. Moreover since

$$
\operatorname{Tr}[(F \pm * F) \wedge(* F \pm F)] \leqq 0
$$

one has:

$$
S(x) \geqq 2 \pi^{2}|K(x)| \forall x \in E^{4} .
$$

From (4.2) and (4.3) it follows that $S \geqq 2 \pi^{2}|K|$ and equality holds only at these configurations for which $F(x)= \pm * F(x) \forall x \in E^{4}$.

This gives an intrinsic way to classify the minima of $S$ and to characterize them as selfdual $\left(F={ }^{*} F\right)$ for antiselfdual $\left(F=-{ }^{*} F\right)$.

Conversely, any selfdual (or antiselfdual) $F$ is a solution of the free Yang-Mills equations $\left(D^{*} F=0\right)$ and is a minimum for $S$ in view of (4.2), (4.3).

We plan in this section to prove that on the lattice the inequality $S_{L} \geqq 2 \pi^{2}\left|K_{L}\right|$ still holds, with the definitions (3.11), (3.12) for $S_{L}$ and $K_{L}$. We shall also characterize on the lattice the "selfdual" and "antiselfdual" solutions.

There will be two major differences, as compared with the continuum case:

a) with the definition (3.12), the Pontrjagin index $K_{L}$ can take any real value;

b) while $S_{L}=2 \pi^{2}\left|K_{L}\right|$ precisely on "antiselfdual" configurations when $K_{L} \leqq 0$, the minimum of $S_{L}(x)$ over the configurations with fixed $K_{L}(x)>0(\forall x)$ is attained for $\mathrm{SU}(2)$ and under suitable conditions, precisely on "selfdual" configurations, but the value of this minimum is strictly larger than $2 \pi^{2} K_{L}(x)$. For a generic $G$, it is moreover much harder to relate "selfdual" configurations with minima of $S_{L}$.

Lemma 4.1. For any pair $P^{\prime}, P^{\prime \prime}$, of complementary plaquettes based at $x$ and for any configuration $g$ we have:

$$
\begin{aligned}
\operatorname{Re}\left(2 \operatorname{Tr} \mathbb{1}-\operatorname{Tr} g\left(P^{\prime}\right)-\operatorname{Tr} g\left(P^{\prime \prime}\right)\right) \geqq & \mid \operatorname{Re}\left(-\operatorname{Tr} \mathbb{1}-\operatorname{Tr}\left(g\left(P^{\prime}\right) g\left(P^{\prime \prime}\right)\right)\right. \\
& \left.+\operatorname{Tr} g\left(P^{\prime}\right)+\operatorname{Tr} g\left(P^{\prime \prime}\right)\right) \mid
\end{aligned}
$$

The equality holds if and only if $g\left(P^{\prime}\right)=\left(g\left(P^{\prime \prime}\right)\right)^{-1}$.

(Recall that we use the same symbols for elements of the group and for representatives in the specific representation which one consider. We shall often write $\mathbb{1}$ instead of $e$.)

Proof. Let $(,)_{\sigma}$ be the inner product of Hilbert-Schmidt in the space of the $m \times m$ matrixes ( $m$ is the dimension of the representation of the group $G$ ).

Then we have:

$$
\operatorname{Re}\left(\operatorname{Tr} \mathbb{1}-\operatorname{Tr} g\left(P^{\prime}\right)\right)=\frac{1}{2}\left\|\mathbb{1}-g\left(P^{\prime}\right)\right\|_{\sigma}^{2}
$$

and

$$
\begin{aligned}
& -\operatorname{Tr} \mathbb{1}-\operatorname{Tr}\left(g\left(P^{\prime}\right) g\left(P^{\prime \prime}\right)\right)+\operatorname{Tr} g\left(P^{\prime}\right)+\operatorname{Tr} g\left(P^{\prime \prime}\right) \\
& \quad=\left(\mathbb{1}-\left(g\left(P^{\prime}\right)\right)^{-1},-\mathbb{1}+g\left(P^{\prime \prime}\right)\right)_{\sigma} .
\end{aligned}
$$


Now for any pair of vectors $x, y$ in an inner product space we have:

$$
|\operatorname{Re}(x, y)| \leqq|(x, y)| \leqq\|x\|\|y\| \leqq \frac{1}{2}\left(\|x\|^{2}+\|y\|^{2}\right) .
$$

So the inequality of the lemma is proved. The equality holds if and only if we have simultaneously:

$$
\begin{aligned}
& \mathbb{1}-\left(g\left(P^{\prime}\right)\right)^{-1}=\lambda\left(\mathbb{1}-g\left(P^{\prime \prime}\right)\right) \text { for some complex } \lambda \\
& \left\|\mathbb{1}-g\left(P^{\prime}\right)\right\|_{\sigma}=\left\|\mathbb{1}-g\left(P^{\prime \prime}\right)\right\|_{\sigma} \\
& \operatorname{Im}\left(\mathbb{1}-\left(g\left(P^{\prime}\right)\right)^{-1}, \mathbb{1}-g\left(P^{\prime \prime}\right)\right)_{\sigma}=0 .
\end{aligned}
$$

That is:

$$
\mathbb{1}-\left(g\left(P^{\prime}\right)\right)^{-1}= \pm\left(\mathbb{1}-g\left(P^{\prime \prime}\right)\right) .
$$

But if we choose the sign minus, then it will be: $2\left(g\left(P^{\prime}\right)+\left(g\left(P^{\prime}\right)\right)^{-1}\right)=4 \mathbb{1}$, which implies $g\left(P^{\prime}\right)=\mathbb{1}$; so the lemma is proved.

Lemma 4.2. For any configuration $g$ and any point $x \in Z_{\varepsilon}^{4}$ we have: $S_{L}(x)$ $\geqq 2 \pi^{2}\left|K_{L}(x)\right|$. The equality holds if and only if $g\left(P^{\prime}(x)\right)=\left(g\left(P^{\prime \prime}(x)\right)\right)^{-1}$ for any pair of complementary plaquettes based at $x$. If the equality holds $K_{L}(x)$ must be $\leqq 0$.

Proof. It is an immediate consequence of Lemma 4.1.

Definition. We shall say that a configuration $g$ on the lattice is antiselfdual at $x$ if $g\left(P^{\prime}(x)\right)=\left(g\left(P^{\prime \prime}(x)\right)\right)^{-1}$ for each pair of complementary plaquettes $P^{\prime}(x), P^{\prime \prime}(x)$ based at $x$. We shall call selfdual at $x$ a configuration $g$ if $g\left(P^{\prime}(x)\right)=g\left(P^{\prime \prime}(x)\right)$ for each pair of complementary plaquettes $P^{\prime}(x), P^{\prime \prime}(x)$ based at $x$. A configuration is called (anti)selfdual if it is (anti)selfdual at each $x \in Z_{\varepsilon}^{4}$.

Remarks. a) Lemma 4.2 says exactly that $S_{L}(x)=2 \pi^{2}\left|K_{L}(x)\right|$ if and only if the configuration is antiselfdual at $x$.

b) For antiselfdual $g$ we have $K_{L}(x) \leqq 0$, while it is not true that $K_{L}(x) \geqq 0$ for all selfdual configurations.

c) In the continuum case for selfdual (or antiselfdual) $F, K=0$ implies $F=0$. On the lattice we may have $K_{L}=0$ also for selfdual configurations which are not trivial. Take for example $G=\mathrm{SU}(2)$ and assume that, for the three biplaquettes based at $x \in Z_{\varepsilon}^{4}, g\left(P^{\prime}(x)\right)=g\left(P^{\prime \prime}(x)\right)=i \sigma_{j}$, where $\sigma_{j}$ is one of the Pauli matrices. Then $K_{L}(x)$ is zero.

d) Unlike the continuum case, in the lattice there may exist configurations which are both selfdual and antiselfdual at a certain point $x$, without being trivial at $x$. [e.g. if $g(P(x)=-\mathbb{1})$ for each plaquette based at $x$ ].

e) The definitions of $K_{L}(x), S_{L}(x)$, selfduality and antiselfduality can be extended also, with no change in terminology, to all 2-cochains on the lattice with values in $G$.

(Recall that in our notation a configuration is a coboundary.)

f) Since 2-cochains can be defined by choosing independently the values of $g(x ; \hat{i}, \hat{j})$ at different points $x \in Z_{\varepsilon}^{4}$, it is obvious that selfdual and antiselfdual cochains will exist for any prefixed value of the Pontrjagin density $K_{L}(x)$ and a fortiori for any fixed value of $K_{L}$. 
That this is possible also within the set of coboundaries is not at all obvious, since a coboundary cannot be constructed assigning the group elements $g(P(x))$ independently at different points in $Z_{\varepsilon}^{4}$. We shall come back to this problem in Sect. 8.

Obviously Lemmas 4.1 and 4.2 hold also for 2-cochains.

g) If we have an antiselfdual (or selfdual) configuration $A$ on the continuum, and we construct a lattice gauge configuration $g^{A}$ via (3.1) and (3.2), we obtain (for $\varepsilon$ small enough):

$$
g^{A}(x ; \hat{i}, \hat{j}) \simeq g_{0}^{A}(x ; \hat{i}, \hat{j})=: \exp \left(\varepsilon^{2} F_{i j}(x)\right) .
$$

Now the 2-cochain $g_{0}^{A}$ is selfdual or antiselfdual depending on whether $A$ is selfdual or antiselfdual.

Proposition 4.3. The action $S_{L}$ considered as a functional on 2-cochains with fixed Pontrjagin number $K_{L} \leqq 0$, attains its minimum on antiselfdual cochains. The minimum value is $S_{L}^{\min }=-2 \pi^{2} K_{L}$.

Proof. Consider a 2-cochain $g$ and let $T_{ \pm}(g)=:\left\{x \mid K_{L}^{g}(x) \gtreqless 0\right\}$. If $T_{+}(g)$ is empty, one can find $a$ 2-cochain $g^{\prime}$ such that $g^{\prime}$ is antiselfdual and

$$
K_{L}^{g}(x)=K_{L}^{g^{\prime}}(x), \quad S_{L}^{g}(x) \geqq S_{L}^{g^{\prime}}(x) \forall x .
$$

If $T_{+}(g)$ is not empty, let $\bar{x} \in T_{+}(g)$.

We construct now a 2-cochain $g^{\prime}$ which is antiselfdual at $T_{-}(g) \cup\{\bar{x}\}$, has the same Pontrjagin index as $g$ and is such that

$$
T_{-}\left(g^{\prime}\right)=T_{-}(g) \cup\{\bar{x}\}, \quad S_{L}^{g^{\prime}} \leqq S_{L}^{g} .
$$

An induction procedure on the points of $T_{+}(g)$ will then complete the proof.

To construct such $g^{\prime}$, notice that from the definition (3.12) one can find a one parameter family of 2-cochains : $g_{\alpha} 0 \leqq \alpha \leqq 1, g_{1}=g, g_{0}(P(x))=\mathbb{1} \forall$ plaquette based at $x$, such that $\left|K_{L}^{g_{\alpha}}(x)\right|$ is a decreasing function of $\alpha$ [with $\left.K_{L}^{g_{0}}(x)=0\right]$. Since

$$
\sum_{x \in T-(g)} K_{L}^{g}(x)<-K_{L}^{g}(\bar{x}) \text {, one can then construct a cochain } g^{\prime} \text { such that }
$$

$$
\begin{aligned}
& K_{L}^{g^{\prime}}(\bar{x})=0, \quad 0 \geqq K_{L}^{g^{\prime}}(x) \geqq K_{L}^{g}(x) \forall x \in T_{-}(g) \\
& K_{L}^{g^{\prime}}(x)=K_{L}^{g}(x) \forall x \in T_{+}(g) \backslash\{\bar{x}\}
\end{aligned}
$$

and such that:

$$
\sum_{x \in T_{-}(g) \cup\{(\bar{x}\}} \mathrm{K}_{L}^{g^{\prime}}(\mathrm{x})=\sum_{x \in T_{-}(g) \cup\{\bar{x}\}} \mathrm{K}_{L}^{g}(\mathrm{x}) .
$$

Proposition 4.4. The action $S_{L}$ considered as a functional on 2-cochains with fixed Pontrjagin number $K_{L} \geqq 0$ satisfies the inequality $S_{L} \geqq 2 \pi^{2} K_{L}$ and the equality holds if and only if $S_{L}=K_{L}=0$ (that is only for trivial configurations).

Proof. Immediate from Lemma 4.2.

In the next section we will study the minima of the action functional for fixed $K_{L}>0$. 
Remark that the definitions of $S_{L}(x), K_{L}(x)$, (anti)selfduality on the lattice can extend also to 2-cochains with values in $U(n)$ : all the results of this section still hold for this extension.

\section{Analysis of the Selfdual Case: SU(2)}

For any 2-cochain $g$ and any biplaquette $B P(x)$ based at $x$ and composed by the two plaquettes $P^{\prime}(x)$ and $P^{\prime \prime}(x)$ we shall write:

$$
\begin{aligned}
& S_{L}(B P(x))=2 \operatorname{Tr} \mathbb{1}-\operatorname{Re}\left(\operatorname{Tr} g\left(P^{\prime}(x)\right)+\operatorname{Tr} g\left(P^{\prime \prime}(x)\right)\right) \\
& K_{L}(B P(x))=\frac{1}{2 \pi^{2}} \operatorname{Re}\left[\operatorname{Tr} g\left(P^{\prime}(x)\right)+\operatorname{Tr} g\left(P^{\prime \prime}(x)\right)-\operatorname{Tr} \mathbb{1}-\operatorname{Tr}\left(g\left(P^{\prime}(x)\right) g\left(P^{\prime \prime}(x)\right)\right)\right] .
\end{aligned}
$$

In this section we want to prove the following:

Proposition 5.1. Let $G$ be $\mathrm{SU}(2)$ in the spinor or vector representation (dim repres. $=2$ or 3 ) and $B$ any finite subset of $Z_{\varepsilon}^{4}$, then:

a) Assign to each $x \in B$ three real numbers $0 \leqq k_{i}(x) \leqq 1 / 2 i=1,2,3$ and consider the set of 2-cochains such that $K_{L}\left(B P_{i}(x)\right)=\frac{1}{\pi^{2}} k_{i}(x) \forall_{i}$ and $\forall x \in B$, and $K_{L}(B P(x))$ $=0 \forall x \notin B$.

The action functional defined on this set of 2-cochains attains its minimum at selfdual cochains; more precisely, given $k_{i}(x)$ as before there exist a unique 2-cochain on which the action reaches the minimum: this cochain must be selfdual.

b) Consider the set $\mathscr{M}\left(K_{L}^{0}, B\right)$ of 2-cochains which are trivial outside of $B$ and have Pontrjagin number $K_{L}=K_{L}^{0}>0$.

(We assume $K_{L}^{0} \leqq 3 N / 2 \pi^{2}$ where $N$ is the number of points of $B$.) The action functional reaches its minimum value on $\mathscr{M}\left(K_{L}^{0}, B\right)$ at selfdual cochains for which $K_{L}(B P(x))$ is independent of $x$ and $B P(x)$. The minimum value for the action is:

$$
S_{L}^{\min }=S_{L}^{\min }\left(K_{L}^{0}\right)=6 N\left(1-\left(1-2 \pi^{2} K_{L}^{0} / 3 N\right)^{1 / 2}\right) .
$$
$K_{L}^{0}>0$.

c) Consider the set $\mathscr{M}\left(K_{L}^{0}\right)$ of cochains which have a prefixed Pontrjagin number

The action functional has on this set of cochains, $2 \pi^{2} K_{L}^{0}$ as the lower bound. The value $2 \pi^{2} K_{L}^{0}$ is not attained on $\mathscr{M}\left(K_{L}^{0}\right)$, i.e. it is not a minimum for $S_{L}$.

Remark. The statement a) says that only a certain subset of the selfdual cochains is the set which minimize the action, once the Pontrjagin index is locally given.

Note that $K_{L}(B P(x))$ cannot be strictly larger than $\frac{1}{2} \pi^{2}$ as we shall see in the proof of Proposition 5.1.

Before passing to the proof of Proposition 5.1 we need one preliminary lemma.

Lemma 5.2. Consider for $\frac{1}{2} \geqq k \geqq 0$ the set $Q_{k} \subset \mathbb{R}^{3}$ defined by:

$$
\begin{aligned}
Q_{k}= & :\{a, b, t \mid-1 \leqq a, b, t \leqq 1 ; \\
& \left.-1-a b+t\left(1-a^{2}\right)^{1 / 2}\left(1-b^{2}\right)^{1 / 2}+a+b=k\right\} .
\end{aligned}
$$


The function $s(a, b, t)=: 2-a-b$ attains its minimum on $Q_{k}$ at a point which satisfies the conditions $a=b ; t=1$.

$$
\begin{aligned}
& s(k)=: \operatorname{Inf}_{Q_{k}} s(a, b, t) \text { is given by: } \\
& s(k)=1-(1-2 k)^{1 / 2} .
\end{aligned}
$$

Proof of the Lemma. Setting $p=(1-a)(1-b) ; s=2-a-b$ one has:

$$
k=-p+t \sqrt{p(4+p-2 s)}
$$

with the constraints :

$$
4 \geqq p \geqq 0 ; \quad 4 \geqq s \geqq 0 ; \quad 2 \geqq s \geqq 2 \sqrt{p} .
$$

Moreover, since $k$ is $\geqq 0$ one has also $t \geqq 0$.

Taking $p$ and $t$ as coordinates in $Q_{k}$, the function $s$ becomes:

$$
s=s_{k}(p, t)=2+p / 2-\left(k^{2} p^{-1}+p+2 k\right) / 2 t^{2} .
$$

We draw now, in the plane $t=\bar{t}$ the allowed region for the parameters $s$ and $p$ and the graph of the curve (5.3) for $\bar{t} \neq 1$ (Fig. 2) and for $\bar{t}=1$ (Fig. 3).

Since $-1-a b+a+b$ is $\leqq 0$, it follows that $t \geqq t_{0}(k)$ for some $t_{0}(k)$ : an easy calculation shows that $t_{0}(k)$ is equal to $\left(k+\left(k^{2}+4 k\right)^{1 / 2}\right) / 2$. From the two graphs it is evident that, for fixed $t$, the minimum of $s$ is attained on the curve $s=2 \sqrt{p}$ i.e. $a=b$; the value of this minimum is :

$$
s_{k}(t)=2\left[t-\left(t^{2}-k(1+t)\right)^{1 / 2}\right] /(1+t) \quad \text { for } \quad t_{0}(k) \leqq t \leqq 1 .
$$

Hence the absolute minimum $s(k)$ on $Q_{k}$ is attained at a point which satisfies the conditions $t=1, a=b$ and (5.1) holds.

Remark. From the condition $t \geqq\left(k+\left(k^{2}+4 k\right)^{1 / 2}\right) / 2$ we deduce also that for $k>1 / 2$, $Q_{k}=\emptyset$.

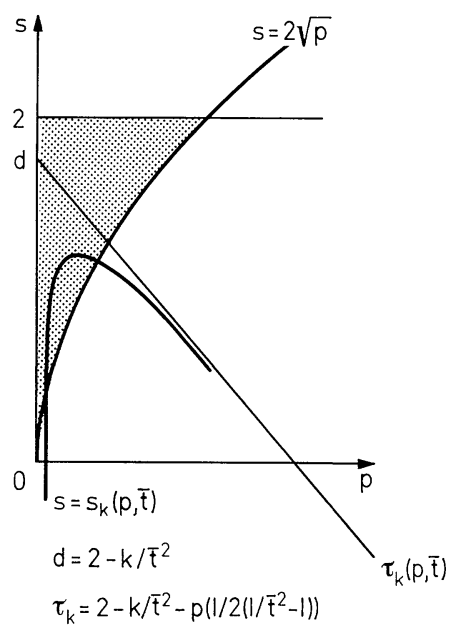

Fig. 2

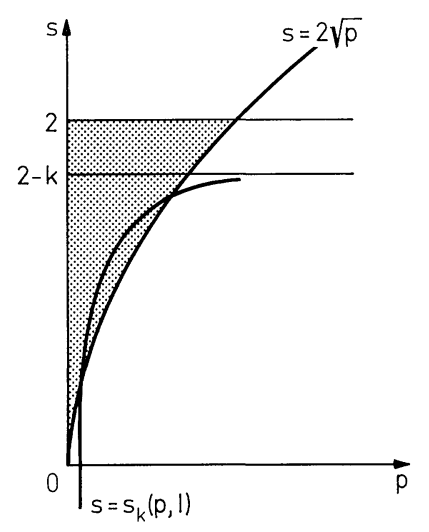

Fig. 3 
It is also evident that in $Q_{k}$ there exist two points with $a=b$ and $t=1$. One of these points satisfies the condition $a \leqq 1 / 2$, while the other has $a \geqq 1 / 2$ and is the point where $s$ reaches its minimum. For the latter point we have $a=\left(1+(1-2 k)^{1 / 2}\right) / 2$ (which goes to 1 as $k \rightarrow 0$ ).

Observe also that when $k \rightarrow 0, s(k)-k=O\left(k^{2}\right)$.

Now we come back to the proof of Proposition 5.1.

Let $\Pi_{m}$ be an irreducibile $m+1$-dimensional representation of $\mathrm{SU}(2)$; $\forall u \in \operatorname{SU}(2)$ there exist an angle $\theta(u)$ and a unit vector $\eta(u) \in \mathbb{R}^{3}$ such that:

$$
\Pi_{m}(u)=\exp [i \theta(u) \boldsymbol{\Sigma} \cdot \boldsymbol{\eta}(u)]
$$

where $\Sigma_{i} i=1,2,3$ are hermitean matrices which satisfy the relations:

$$
\left[\Sigma_{i}, \Sigma_{j}\right]=\sqrt{-1} \varepsilon_{i j k} \Sigma_{k} .
$$

The eigenvalues of each $\Sigma_{i}$ and of $\boldsymbol{\Sigma} \cdot \boldsymbol{\eta}$ are $(\forall \boldsymbol{\eta})$ the numbers :

$$
-m / 2 ;-m / 2+1 ; \ldots ; m / 2-1 ; m / 2 \text {. }
$$

One has therefore: $\operatorname{Tr}(\boldsymbol{\Sigma} \cdot \boldsymbol{\eta})^{n}=\sum_{p=0}^{m}(-m / 2+p)^{n}$, and, using (5.5):

$$
\begin{aligned}
\operatorname{Tr} \Pi_{m}(u) & =\sum_{n=0}(\sqrt{-1})^{n} / n ! \theta^{n}(u) \operatorname{Tr}(\Sigma \cdot \eta(u))^{n} \\
& =\sum_{p=0}^{m} \cos (\theta(u)(-m / 2+p))
\end{aligned}
$$

For every irreducible representation one has moreover:

$$
\exp \left(\sqrt{-1} \theta_{1}\left(\boldsymbol{\Sigma} \cdot \boldsymbol{\eta}_{1}\right)\right) \exp \left(\sqrt{-1} \theta_{2}\left(\boldsymbol{\Sigma} \cdot \boldsymbol{\eta}_{2}\right)\right)=\exp \left(\sqrt{-1} \theta_{12}\left(\boldsymbol{\Sigma} \cdot \boldsymbol{\eta}_{12}\right)\right),
$$

where, setting $t=: \boldsymbol{\eta}_{1} \cdot \boldsymbol{\eta}_{2}$

$$
\cos \left(\theta_{12} / 2\right)=\cos \left(\theta_{1} / 2\right) \cos \left(\theta_{2} / 2\right)-t \sin \left(\theta_{1} / 2\right) \sin \left(\theta_{2} / 2\right) .
$$

If we have now on the lattice $Z_{\varepsilon}^{4}$ a 2 -cochain with values in $\Pi_{m}(\mathrm{SU}(2))$, the action and the Pontrjagin number calculated at any biplaquette $B P(x)$ are given by:

$$
\begin{aligned}
S_{L}(B P(x))= & 2(m+1)-\sum_{p=0}^{m} \cos \left[(-m / 2+p) \theta\left(P^{\prime}(x)\right)\right] \\
& +-\sum_{p=0}^{m} \cos \left[(-m / 2+p) \theta\left(P^{\prime \prime}(x)\right)\right] \\
K_{L}(B P(x))= & \frac{1}{2 \pi^{2}}\left\{-(m+1)+\sum_{p=0}^{m} \cos \left[(-m / 2+p) \theta\left(P^{\prime}(x)\right)\right]\right. \\
& +\sum_{p=0}^{m} \cos \left[(-m / 2+p) \theta\left(P^{\prime \prime}(x)\right)\right] \\
& \left.-\sum_{p=0}^{m} \cos \left[(-m / 2+p) \theta_{12}(B P(x))\right]\right\} .
\end{aligned}
$$


If $m=1$, that is if we have the spinor representation, (5.6)-(5.10) together with Lemma 5.2 give immediately the proof of the Part a) of Proposition 5.1.

[We set $a=\cos \left(\theta\left(P^{\prime}(x)\right) / 2\right) ; b=\cos \left(\theta\left(P^{\prime \prime}(x)\right) / 2\right) ; s=S_{L}(B P(x)) / 2 ; k=\pi^{2} K_{L}(B P(x))$ and $t$ defined as in (5.8).]

If $m=2$ (vector representation):

$$
\begin{aligned}
& K_{L}(B P(x))= \frac{1}{\pi^{2}}\left\{-1+\cos \theta\left(P^{\prime}(x)\right)+\cos \theta\left(P^{\prime \prime}(x)\right)\right. \\
&\left.-\cos \theta_{12}(B P(x))\right\}, \\
& S_{L}(B P(x))=2\left(2-\cos \theta\left(P^{\prime}(x)\right)-\cos \theta\left(P^{\prime \prime}(x)\right)\right) .
\end{aligned}
$$

Setting once again :

$$
\begin{aligned}
& s=S_{L}(B P(x)) / 2 ; \quad a=\cos \theta\left(P^{\prime}(x)\right) ; \quad b=\cos \theta\left(P^{\prime \prime}(x)\right), \\
& p=(1-a)(1-b) ; \quad k=\pi^{2} K_{L}(B P(x)) ;
\end{aligned}
$$

$t$ defined as in (5.8), one has ${ }^{1}$ :

$$
k=-p\left(1+t^{2}\right) / 2+t \sqrt{p(4+p-2 s)},
$$

that is:

$$
s=s_{k}(p, t)=2+p / 2-\left(k+p\left(1+t^{2}\right) / 2\right)^{2} / 2 p t^{2} .
$$

Now we can repeat the discussion of Lemma 5.2, show that all conclusions still hold [including Eq. (5.1)] and hence prove the Part a) of Proposition 5.1 also for vector representations of $\mathrm{SU}(2)$, provided that $\partial_{t} s_{k}(p, t)>0$ for $s$ given by (5.12). But this is obviously true, since:

$$
\partial_{t} s=\left(k+\left(1+t^{2}\right) p / 2\right)\left(k+\left(1-t^{2}\right) p / 2\right) / p t^{3}>0 .
$$

It should be possible to prove in the same way something like Part a) of Proposition 5.1 for any representation of $\mathrm{SU}(2)$.

To prove statements b) and c) of Proposition (5.1), observe firstly that from Eq. (5.1) we have:

$$
s\left(\left(k_{1}+k_{2}\right) / 2\right) \leqq\left(s\left(k_{1}\right)+s\left(k_{2}\right)\right) / 2 \text { for any } 1 / 2 \geqq k_{1}, k_{2} \geqq 0 .
$$

That is if we have two 2-cochains $g$ and $g^{\prime}$ which minimize the action in the sense of Part a) of Proposition 5.1 and are such that for some biplaquettes $B P(x)$ and $B P(y)$ :

$$
K_{L}^{g^{\prime}}(B P(x))=K_{L}^{g^{\prime}}(B P(y))=\left(K_{L}^{g}(B P(x))+K_{L}^{g}(B P(y))\right) / 2,
$$

then:

$$
S_{L}^{g^{\prime}}(B P(x))+S_{L}^{g^{\prime}}(B P(y)) \leqq S_{L}^{g}(B P(x))+S_{L}^{g}(B P(y)) .
$$

Now considering the fact that $s(k)$ is an increasing function of $k$, we can repeat arguments similar to those used in Proposition 4.3 and obtain $b$ ).

Statement c) is an easy consequence of b) and Proposition 4.4.

1 We use the identity:

$\cos \theta_{12}=\cos \theta_{1} \cos \theta_{2}-t \sin \theta_{1} \sin \theta_{2}-2\left(1-t^{2}\right)\left(1-\cos ^{2} \theta_{1} / 2\right)\left(1-\cos ^{2} \theta_{2} / 2\right)$ 


\section{Selfdual Case; General Results for any (Non Abelian, Simple, Compact Lie) Group $G$}

For a general group $G$ we are not able to find results similar to those obtained in Sect. 5 for SU(2).

We can only show that, if we consider 2-cochains with values in a suitable neighborhood of the identity of $G$, then selfdual cochains minimize the action in an approximate sense, for (locally) fixed Pontrjagin index.

For simplicity we shall consider only representations with real character. Let now $g$ be a 2-cochain and $m$ be the dimension of the considered representation of $G$; we set for each biplaquette $B P(x)=\left\{P^{\prime}(x), P^{\prime \prime}(x)\right\}$ :

$$
\begin{array}{ll}
a=\left(\mathbb{1}, g\left(P^{\prime}\right)\right)_{\sigma} / m & a_{n}=-i\left(A_{n}, g\left(P^{\prime}\right)\right)_{\sigma} / m^{1 / 2} \\
b=\left(\mathbb{1}, g\left(P^{\prime \prime}\right)\right)_{\sigma} / m & b_{n}=-i\left(A_{n}, g\left(P^{\prime \prime}\right)\right)_{\sigma} / m^{1 / 2},
\end{array}
$$

where $(,)_{\sigma}$ is the Hilbert-Schmidt inner product, $A_{n} n=1, \ldots, m^{2}-1$ are hermitean, form an orthonormal system and satisfy the condition $\operatorname{Tr} A_{n}=0 \forall n$.

In this notation, the action and the Pontrjagin number relative to the biplaquette $B P(x)$ are :

$$
\begin{aligned}
S_{L}(B P(x))=m s, \quad \text { where we have set } s= & 2-a-b ; \\
K_{L}(B P(x))=m k / 2 \pi^{2}, \text { where we have set } k= & 1-a b+t\left(1-a^{2}\right)^{1 / 2}\left(1-b^{2}\right)^{1 / 2} \\
& -(2-a-b)
\end{aligned}
$$

with :

$$
t=\sum_{n=1}^{m^{2}-1} a_{n} b_{n}\left(1-a^{2}\right)^{-1 / 2}\left(1-b^{2}\right)^{-1 / 2} .
$$

Since we are dealing only with representations with real character, $a, b, t$ are real and moreover $|t| \leqq 1$.

We assume $k$ fixed $>0$.

If $q$ is the dimension of $\mathrm{g}^{2}$, we can choose the $A_{n}$ in (6.1) in such a way that $\left\{i A_{n}\right\}$ for $n=1, \ldots, q$ is a basis for (the representation we are considering of $\mathfrak{g}$. This is possible since, if $m$ is the dimension of the representation we are considering, then $\mathfrak{g}$ is a linear subspace of the Lie algebra of $\mathrm{SU}(m)$.

In this case $a_{n}, b_{n}$ are real for $1 \leqq n \leqq q$; indeed for such $n$ we have:

$$
\begin{aligned}
a_{n} & =-\operatorname{Tr}\left(i A_{n} g\left(P^{\prime}\right)\right) / m^{1 / 2} \\
& \left.=-\operatorname{Tr} \lim _{a \rightarrow 0} g\left(P^{\prime}\right)\left(g^{n}(\alpha)-\mathbb{1}\right) / \alpha\right) / m^{1 / 2} \\
& =-\lim _{\alpha \rightarrow 0} \operatorname{Tr}\left(g^{n}(\alpha) g\left(P^{\prime}\right)-g\left(P^{\prime}\right)\right) / \alpha m^{1 / 2},
\end{aligned}
$$

where $\alpha \rightarrow g^{n}(\alpha)$ is the one parameter subgroup generated by $A_{n}$.

Now from Lemma 5.2 we have:

$$
t \geqq t_{0}(k) \text { for some } t_{0}(k) \text {; }
$$

2 As a vector space 
moreover, since not all $a_{n}, b_{n}$ are (in general) real, we have also:

$$
t \leqq t^{\max }(k) \leqq 1
$$

where $t^{\max }(k)$ is the 1.u.b. of all $t$ expressed by (6.4) when we consider all 2-cochains subjected to the condition that $K_{L}(B P(x))$ satisfies the (6.3) (remember that $k$ is fixed).

If we take now a selfdual assignment (i.e. $a=b ; a_{n}=b_{n} \forall n$ ) we have, for such $g\left(P^{\prime}(x)\right)$ :

$$
t=t_{\text {s.d. }}=\sum_{n=1}^{m^{2}-1}\left(a_{n}\right)^{2} / \sum_{n=1}^{m^{2}-1}\left|a_{n}\right|^{2} .
$$

From the discussion in Lemma 5.2 it follows that the minimum for $s$ should be attained, once $k>0$ is fixed, when $a=b$ and $t$ is the largest value compatible with (6.7).

Actually we do not know even if there exist a cochain which satisfies (6.3) and verifies the conditions $t=t^{\max }(k), a=b$.

In any case we shall call $\bar{Q}_{k}$ the subset of $\mathbb{R}^{3}$ defined as in Lemma 5.2 with the additional restriction (6.7) and :

$s(k)=: \operatorname{Inf}_{\bar{Q}_{k}} \mathrm{~s}(\mathrm{a}, \mathrm{b}, \mathrm{t}) \quad$ with, as usual $s(a, b, t)=: 2-a-b$.

We conjecture also that (in general) $t^{\max }(k)$ is not of the form (6.8). But the situation is more favorable if we consider only cochains with values in a suitable neighborhood of the identity of $G$ (the size of this neighborhood depends on $G$ and on the representation considered).

Then we have:

Proposition 6.1. Let $s(k)$ be defined as in (6.9). Then if we have a selfdual cochain $g$ which satisfies (6.3) it must be:

$$
s_{\mathrm{s} . \mathrm{d} .}-s(k)=O\left(s(k)^{2}\right), \quad \text { where we have set } s_{\mathrm{s.d} .}=: S_{L}^{g}(B P(x)) / 2 \text {. }
$$

Proof. For small $s(k)$ (and hence for small $k$ ), we can consider only 2-cochains $\tilde{g}$ such that $\tilde{g}\left(P^{\prime}\right)$ and $\tilde{g}\left(P^{\prime \prime}\right)$ are in a suitable neighborhood $U_{k}$ of the identity of $G$.

That is we can set:

$$
\begin{aligned}
& \tilde{g}\left(P^{\prime}\right)=\mathbb{1}+\alpha_{k} A\left(P^{\prime}\right)+O\left(\alpha_{k}^{2}\right) \\
& \tilde{g}\left(P^{\prime \prime}\right)=\mathbb{1}+\alpha_{k} A\left(P^{\prime \prime}\right)+O\left(\alpha_{k}^{2}\right)
\end{aligned}
$$

with $\left\|A\left(P^{\prime}\right)\right\|_{\sigma} \leqq 1,\left\|A\left(P^{\prime \prime}\right)\right\|_{\sigma} \leqq 1\left(\alpha_{k}\right.$ represents the size of $\left.U_{k}\right)$.

Since $\sup _{\tilde{g}\left(P^{\prime}\right), \tilde{g}\left(P^{\prime \prime}\right) \in U_{k}}\{s\} \sim \alpha_{k}^{2}$, we can assume $\alpha_{k}^{2} \sim s(k)$.

For $\tilde{g}\left(P^{\prime}\right), \tilde{g}\left(P^{\prime \prime}\right) \in U_{k}$ we have:

$$
t=\sum_{n=1}^{q} a_{n} b_{n}\left(1-a^{2}\right)^{-1 / 2}\left(1-b^{2}\right)^{-1 / 2}+O\left(\alpha_{k}^{2}\right)
$$

and for our selfdual $g: t_{\text {s.d. }}=1+O\left(\alpha_{k}^{2}\right)$, therefore it must be $t^{\max }(k)=1+O\left(\alpha_{k}^{2}\right)$, but this implies : $t_{\text {s.d. }}-t^{\max }(k)=O(s(k))$, so, taking into account (5.4), one has :

$$
\begin{aligned}
& s(k)=k / t^{\max }(k)+O\left(s(k)^{2}\right) \\
& s_{\text {s.d. }}=k / t_{\text {s.d. }}+O\left(s(k)^{2}\right) ;
\end{aligned}
$$


therefore:

$$
\begin{aligned}
s_{\text {s.d. }}-s(k) & =k / t_{\text {s.d. }}-k / t^{\max }(k)+O\left(s(k)^{2}\right) \\
& =k\left(t^{\max }(k)-t_{\text {s.d. }}\right) / t_{\text {s.d. }} t^{\max }(k)+O\left(s(k)^{2}\right)=O\left(s(k)^{2}\right) .
\end{aligned}
$$

\section{A Possible New Definition of the "Pontrjagin Index" on the Lattice}

Let $x \in Z_{\varepsilon}^{4}, x=\left(n_{1} \varepsilon, n_{2} \varepsilon, n_{3} \varepsilon, n_{4} \varepsilon\right)$ and let $\sigma_{12}: Z_{\varepsilon}^{4} \rightarrow Z_{\varepsilon}^{4}$ be defined as follows:

$$
\sigma_{12}(x)=:\left(n_{2} \varepsilon, n_{1} \varepsilon, n_{3} \varepsilon, n_{4} \varepsilon\right) \text {. }
$$

The mapping $\sigma_{12}$ corresponds to the inversion of the coordinated axes $\hat{1}$ and $\hat{2}$, and can easily extend to 1- and 2-cochains (with values in any non abelian, simple, compact Lie group $G$ ):

$$
\begin{aligned}
& \sigma_{12}(f(x ; \hat{j}))=: f\left(\sigma_{12} x ; \sigma_{12}(\hat{j})\right) \\
& \sigma_{12}\left(g(x ; \hat{i}, \hat{j})=: g\left(\sigma_{12} x ; \sigma_{12}(\hat{i}), \sigma_{12}(\hat{j})\right),\right.
\end{aligned}
$$

where we have set:

$$
\sigma_{12}(\hat{1})=\hat{2} ; \sigma_{12}(\hat{2})=\hat{1} ; \sigma_{12}(\hat{3})=\hat{3} ; \sigma_{12}(\hat{4})=\hat{4} .
$$

In the same way we can consider mappings $\sigma_{i j}$ for any pair of directions $\hat{i}$ and $\hat{j}$.

For mappings $\sigma_{i j}$ we have the following properties:

a) $\sigma_{i j}$ transforms selfdual 2-cochains (or configurations) in antiselfdual 2cochains (or configurations) and viceversa.

In particular this implies that in $Z_{\varepsilon}^{4}$ we have as many selfdual configurations as antiselfdual configurations.

b) for any 2-cochain $g$ with action $S_{L}(g)$, we have:

$S_{L}(g)=S_{L}\left(\sigma_{i j} g\right)$.

c) Let $g$ be any 2-cochain with Pontrjagin index $K_{L}(g)$; in general it is not true that $K_{L}(g)=-K_{L}\left(\sigma_{i j} g\right)$.

Indeed consider a 2-cochain which is selfdual at some $B P(x)$ and for which $K_{L}(B P(x))<0$ [e.g. if $\left.g\left(P^{\prime}(x)\right]=g\left(P^{\prime \prime}(x)\right)=-\mathbb{1}\right)$. The mapping $\sigma_{i j}$ transforms the biplaquette $B P(x)$ in another biplaquette $\sigma_{i j}(B P(x))$; the configuration $\sigma_{i j}(g)$ is antiselfdual at $\sigma_{i j}(B P(x))$ and so $K_{L}\left(\sigma_{i j}(g)\right)$ calculated at $\sigma_{i j}(B P(x))$ is also negative. This example shows also that in general it is not even true that $K_{L}(g) \leqq 0$ implies $K_{L}\left(\sigma_{i j} g\right) \geqq 0$.

$$
\begin{aligned}
& \text { d) For any 2-cochain } g \text { and any } \sigma_{i j}, \sigma_{m n} \text { we have: } \\
& K_{L}\left(\sigma_{i j}\left(\sigma_{m n}(g)\right)\right)=K_{L}(g) \text {. }
\end{aligned}
$$

This can be easily proved, using the fact that for unitary matrices $U, V \operatorname{Tr} U V$ $=\operatorname{Tr} V U$ and $\operatorname{Re} \operatorname{Tr} U=\operatorname{Re} \operatorname{Tr} U^{-1}$.

Now define for any 2-cochain $g$ 
We may consider the possibility of assuming $\bar{K}_{L}$ as a new definition of the "Pontrjagin index" on the lattice.

Observe that:

a) The definition (7.6) is independent of the choice of $\sigma_{i j}$. This is a direct consequence of (7.5).

b) $\bar{K}_{L}(g)$ is in general different from $-\overline{\mathrm{K}}_{L}\left(\sigma_{i j} \mathrm{~g}\right)$. Indeed $\overline{\mathrm{K}}_{L}(\mathrm{~g})$ is different from $-\overline{\mathrm{K}}_{L}\left(\sigma_{i j} \mathrm{~g}\right)$ for all 2-cochains which satisfy the condition $K_{L}(g)<0$ and $K_{L}\left(\sigma_{i j} g\right)<0$.

On the continuum, if we change the orientation of the base manifold, the Pontrjagin index is transformed in its opposite, so we can deduce that the asymmetry we have discovered is, in a certain sense, "intrinsic" of the lattice.

c) $K_{L}(g)=0$ does not imply $K_{L}\left(\sigma_{i j} g\right)=0$. Take once again for example, a 2cochain with values in $\mathrm{SU}(2)$ such that for every biplaquette it is $g\left(P^{\prime}\right)=g\left(P^{\prime \prime}\right)=i$ times one of the three Pauli matrices.

This shows also that $\bar{K}_{L}$ considered as a function from the space of all 2cochains (with support contained in a fixed finite hypercubical lattice) into $\mathbb{R}$ is not continuous; the points of discontinuity are 2-cochains with zero Pontrjagin index.

d) Let $A$ be a configuration on the continuum (with Pontrjagin index $K$ ) and let $A^{\sigma}$ be the configuration obtained changing the orientation of the base manifold (e.g. $A_{\mu} \rightarrow A_{\sigma_{12} \mu} ; F_{\mu, v} \rightarrow F_{\sigma_{12} \mu, \sigma_{12} v}$ ). Then if $g^{A}$ is the lattice configuration obtained as in (3.1), (3.2), we have $g^{A}=\sigma_{12}\left(g^{A^{\sigma}}\right)$, so we can say that also $\bar{K}_{L} \rightarrow K$ when the lattice spacing goes to zero.

e) $S_{L} \geqq 2 \pi^{2}\left|\bar{K}_{L}\right|$.

This is an immediate consequence of (7.4).

f) $S_{L}(g)=-2 \pi^{2} \bar{K}_{L}(g)$ if and only if the 2-cochain $g$ is antiselfdual.

Indeed $\bar{K}_{L}(g)$ is negative either when $K_{L}(g)$ is negative, or when $K_{L}(g)$ and $K_{L}\left(\sigma_{i j} g\right)$ are both positive.

But if there exist non trivial cochains which satisfy the second condition, then by (7.4) and Proposition 4.4 they cannot satisfy the equality $S_{L}=-2 \pi^{2} \bar{K}_{L}$.

g) $S_{L}(g)=2 \pi^{2} \bar{K}_{L}(g)$ if and only if the 2-cochain $g$ is selfdual and satisfy the condition $K_{L}(g)>0$ (unless $g$ is trivial).

Remark that a selfdual cochain with values in $\mathrm{SU}(2)$ (spinor representation) satisfies the required condition if $\operatorname{Tr} g(P)>0$ for each plaquette $P$.

More generally if we have a selfdual 2-cochain with values in a suitable neighborhood of the identity of $G$, then, setting: $g(P)=: \mathbb{1}+\alpha A(P)+\frac{\alpha^{2}}{2} A^{2}(P)$ $+O\left(\alpha^{3}\right)$ with $A(P) \in \mathfrak{g},\|A(P)\|_{\sigma} \leqq 1$, we have:

$$
2 \pi^{2} K_{L}(B P)=-\alpha^{2} \operatorname{Tr} A^{2}(P)+O\left(\alpha^{3}\right)>0
$$

for sufficiently small $\alpha$ (e.g. $\alpha<1 / 2)$.

h) We can also define a new "Pontrjagin density" setting $\forall x \in Z_{\varepsilon}^{4}$ and for each cochain $g$

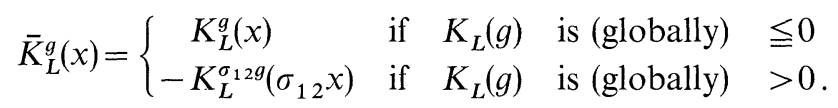




\section{On the Existence of (Anti)selfdual Configurations}

If we have a 2-cochain $g$, the problem of determining whether $g$ is a coboundary or not is in general a very difficult problem: we have to solve (an infinite number of) equations in which the group element associated to a link which belongs to a plaquette based at $x=\left(n_{1} \varepsilon, n_{2} \varepsilon, n_{3} \varepsilon, n_{4} \varepsilon\right)$ depends on all the group elements associated to the links which belong to the plaquettes based at each $y=\left(k_{1} \varepsilon, k_{2} \varepsilon\right.$, $k_{3} \varepsilon, k_{4} \varepsilon$ ) with $k_{i} \leqq n_{i} \forall i$.

Remark that also in the continuum case if we have a 2 -form $F$ on the base manifold $M$ with values in the Lie algebra $\mathfrak{g}$, the problem of determining whether $F$ essentially arises from the curvature form of a $G$-principal bundle over $M$ with a suitable connection form $\omega$ or not, is a problem which is far from being solved.

On the lattice, if we consider cochains with values in an abelian group, then the problem can be dealt with in the context of cubical cohomology $([14,15])$. Since this cohomology is equivalent to the singular cohomology and since we deal with $E^{4}$, then a 2-cochain is a coboundary if and only if it is a cocycle.

For non abelian groups, we start considering a fixed (finite) hypercubical sublattice $\Lambda$ of $Z_{\varepsilon}^{4}$ with $N^{4}$ points and we proceed as follows: we fix the origin of the coordinated axes at the "lowest" point of $\Lambda$, and, starting from the point $(0,0,0,0)$, we assign to each point $x \in \Lambda$ the group elements associated to the 16 links drawn in Fig. 1.

Let $\Lambda_{n}$ be the hypercube $\left\{\left\{m_{i} \varepsilon\right\} \mid \max _{i} m_{i} \leqq n\right\}$; we assign elements of $G$ to the links of the biplaquettes based at $\left\{m_{i} \varepsilon\right\}$ in an order for which points in $\Lambda_{n} \backslash \Lambda_{n-1}$ come before points in $\Lambda_{n+1} \backslash \Lambda_{n}$. At the point $\left\{m_{i} \varepsilon\right\}$ make arbitrary assignments to the links not previously marked, and guarantee (anti)selfduality by a proper choice of $g\left(\left\{p_{i} \varepsilon\right\}+\hat{k} ; \hat{s}\right), s \neq k$ if $p_{k} \geqq p_{i} \forall i$ (so that $\left\{p_{i} \varepsilon\right\} \in \Lambda_{p_{k}} \backslash \Lambda_{p_{k}-1}$ ). To prove consistency, one checks that the latter three links were not already marked. Indeed, $\left\{p_{i} \varepsilon\right\}$ $+\hat{k} \in \Lambda_{p_{k}+1}$, and on the other hand if $\left\{q_{i} \varepsilon\right\} \in \Lambda_{q_{h}} \backslash \Lambda_{q_{h}-1}$ is such that $\left\{q_{i} \varepsilon\right\}+\hat{h}=\left\{p_{i} \varepsilon\right\}$ $+\hat{k}$, then $h=k$ and $p_{i}=q_{i} \forall i$.

It is easy to see that for large $N$ the space of all configurations which are (anti)selfdual at each point of $\Lambda$ (but not on the boundary of $\Lambda$ ) is $G^{N^{4}}$, while the space of all configurations and of all 2-cochains defined on $\Lambda$ is, respectively $G^{4 N^{4}}$ and $G^{6 N^{4}}$.

So, on a finite lattice, we are not able to find general conditions under which a (anti)selfdual 2-cochain is a coboundary, but we can say that there exist (sufficiently many) configurations which are (anti)selfdual everywhere except the boundary.

The situation is much more difficult on a infinite lattice: it is hard to give a general procedure to construct (anti)selfdual configurations with finite action.

Anyway the following considerations may be useful.

Let $\Xi_{N}$ be the set of points of $Z_{\varepsilon}^{4}$ whose coordinates are $\left(n_{1} \varepsilon, n_{2} \varepsilon, n_{3} \varepsilon, n_{4} \varepsilon\right)$ with $n_{i}>N$ and for any 2-cochain $g$ defined on $Z_{\varepsilon}^{4}$ let $S_{N}(g)=: \sum_{x \in \Xi_{N}} S_{L}^{g}(x)$ and $K_{N}(g)=: \sum_{x \in \Xi_{N}} K_{L}^{g}(x)$

If $g$ has finite action $S_{N} \underset{N \rightarrow \infty}{\longrightarrow} 0$ and we can choose $N$ large enough to set $\forall x \in \Xi_{N}$ and $\forall$ plaquette $(x ; \hat{\mu}, \hat{v})$ :

$$
g(x ; \hat{\mu}, \hat{v})=\exp \left(A_{\mu \nu}(x)\right) \quad \text { with } \quad A_{\mu \nu}(x) \in \mathfrak{g} .
$$


If we are able to assign to every $\operatorname{link}(x ; \hat{\mu}), \mu=1,2,3,4, x \in \Xi_{N}$ an element $A_{\mu}(x) \in \mathfrak{g}$ such that:

$$
A_{\mu v}(x)=A_{\mu}(x)+A_{v}(x+\hat{\mu})-A_{\mu}(x+\hat{v})-A_{v}(x),
$$

then if $f$ is defined by:

$$
f:(x ; \hat{\mu}) \rightarrow \exp \left(A_{\mu}(x)\right),
$$

the coboundary $g^{\prime}=: d_{L} f$ defined on $\Xi_{N}$ satisfies the conditions :

$$
S_{N}(g)-S_{N}\left(g^{\prime}\right)=o\left(S_{N}(g)\right) ; \quad K_{N}(g)-K_{N}\left(g^{\prime}\right)=o\left(S_{N}(g)\right) .
$$

So if we have a 2-cochain $g$ with finite action, the problem of finding a coboundary which is a good approximation of $g$ outside a suitable hypercube is a problem of abelian cohomology.

In particular if $g$ is selfdual, then we can construct a 2-coboundary $g^{\prime}=d_{L} f$, where $f$ is given by (8.3) and (8.4), if and only if $\forall x \in \Xi_{N}$ we have:

$$
\left.\begin{array}{rl}
A_{13}(x)+A_{14}(x+\hat{1})+A_{12}(x+\hat{3}) & =A_{13}(x+\hat{2})+A_{14}(x)+A_{12}(x) \\
A_{14}(x)+A_{13}(x)+A_{12}(x+\hat{4}) & =A_{13}(x+\hat{1})+A_{14}(x+\hat{2})+A_{12}(x) \\
A_{13}(x)+A_{12}(x)+A_{14}(x+\hat{3}) & =A_{13}(x+\hat{4})+A_{14}(x)+A_{12}(x+\hat{1}) \\
A_{12}(x)+A_{13}(x)+A_{14}(x)=A_{12}(x+\hat{2})+A_{13}(x+\hat{3})+A_{14}(x+\hat{4})
\end{array}\right\}
$$

where $A_{\mu v}(x)$ are defined as in (8.1).

In conclusion, given a finite hypercubical lattice, we are able to construct all the configurations which are selfdual or antiselfdual at all the points of the hypercube, except the boundary; outside we are able to construct "quasi-selfdual" or "quasi-antiselfdual" configurations which are good approximations of any (anti)selfdual 2-cochain $g$, provided that:

a) $g$ has finite action (or more precisely $S_{N}(g)$ decreases fast enough);

b) $g$ satisfies conditions $(8.5)^{3}$.

The problem which is still open is obviously the problem of doing a right soldering at the boundary, i.e. the problem of controlling the behaviour of our configurations at the edge of the chosen hypercube: we plan to come back to this argument in a future publication.

Acknowledgements. We would like to thank M. Forger, J. E. Roberts, R. Schrader, R. Stora for useful discussions and comments. One of us (P.C.R.) would also like to thank R. Cirelli for warm support and encouragement and R. Schrader for the hospitality at the Institut fur theoretische Physik, Freie Universität Berlin, during the Academic year 1978/1979.

\section{References}

1. Atiyah, M.F., Hitchin, N.J., Singer, I.M. : Proc. R. Soc. Lond. A 362, 425-461 (1978)

2. Atiyah, M.F., Drinfeld, V.G., Hitchin, N.J., Manin, Yu.I. : Phys. Lett. 65 A, 185-187 (1978)

3. Atiyah, M.F., Ward, R.S.: Commun. Math. Phys. 55, 117-124 (1977)

4. Stora, R.: Lecture notes (Erice 1977). In: Invariant wave equations, G. Velo, A.S. Wightman (eds.). Berlin, Heidelberg, New York: Springer 1978

3 In the antiselfdual case the conditions are slightly different 
5. Schwarz, A.S. : Phys. Lett. 67 B, 172-174 (1977)

6. Schwarz, A.S.: Commun. Math. Phys. 64, 233-268 (1979)

7. Mayer, M.E.: Lecture notes (Bonn 1977). In: Differential geometrical methods in mathematical physics. II. K. Bleuler, H. Petry, A. Reetz (eds.). Berlin, Heidelberg, New York: Springer 1978

8. Coleman, S.: The uses of instantons. Lectures delivered at the Intern. School of Subnuclear Physics (Erice 1977) (to be published)

9. Frölich, J.: In : Mathematical problems in theoretical physics. G. Dell'Antonio, S. Doplicher, G. Jona-Lasinio (eds.). Berlin, Heidelberg, New York: Springer 1978

10. Brydges, D., Fröhlich, J., Seiler, E. : preprint, I.H.E.S. (Bures-sur-Yvette) 1978

11. Wilson, K.G.: Phys. Rev. D 10, 2445-2459 (1974)

12. Balian, R., Drouffe, J.M., Itzykson, C. : Phys. Rev. D 10, 3376-3395 (1974); Phys. Rev. D 11, 20982103 (1975); Phys. Rev. D 11, 2104-2119 (1975)

13. Osterwalder, K., Seiler, E.: Ann. Phys. 110, 440-471 (1978)

14. Eilenberg, S., MacLane, S.: Am. J. Math. 75, 189-199 (1953)

15. Hu. S.T.: Homotopy theory. New York, London: Academic Press 1959

Communicated by K. Osterwalder

Received June 5, 1979 
